\title{
Stability assessment methods of technological processes
}

\author{
Julius Tchigirinsky ${ }^{1, ~ *}$, Nataly Chigirinskaya ${ }^{2}$, and Alexander Evtyunin ${ }^{1}$ \\ ${ }^{1}$ Volgograd State Technical University, Technology of Machinery Department, 400005 Volgograd, \\ Russia \\ ${ }^{2}$ Volgograd State Technical University, High Mathematics Department, 400005 Volgograd, Russia
}

\begin{abstract}
Ensuring the stability of quality indicators and product accuracy in modern conditions requires not only classical production measures but also organizational arrangements, which deals with clarifying and modernizing the conceptual apparatus. The study analyzes the methods for assessing the stability of machining, established in the standard process documents regulating the issues of product quality management in mechanical engineering, and reveals the ambiguity of the term 'stability' relating to processes under transition to the arrangement of digital production and the use of equipment with adaptive control. The Fisher criterion can be used for quantitatively analyzing the stability of processes under statistical control.
\end{abstract}

\section{Basic concepts}

In this study, we consider the possibility of quantitatively assessing the stability of the processes that form a complex array of product quality indicators in relation to the products of engineering and machining in particular. We believe that the result of production should be associated not with a product itself - i.e, a separate part, assembly unit, machine - but with a product that has a set of mainly quantitative characteristics that determine the consumer properties of the product. With regard to machining processes, the concept of quality includes "... a set of properties (products) that determine their suitability to fulfill their functional purpose in a given range of changes in operating conditions" $[1,2$, etc]. In this interpretation, 'quality' as the degree of conformity of product parameters to specified requirements [3] is important not for each single product but for the entire production program. Thus, one should consider the constancy of ensuring the stability of production results over a certain time interval, the duration of which can specified, e.g., by the period of the tool life.

To define the concept of stability, the regulatory documentation currently uses several different terms, which, at first glance, are similar in meaning:

- stability is "a property of a technological process that determines the constancy of the probability distributions of its parameters over a certain time interval without outside interference" $[4,5]$, - stability coefficient (1):

\footnotetext{
* Corresponding author: Julio-Tchigirinsky@yandex.ru
} 


$$
k_{S}=\frac{S_{t 1}}{S_{t 2}}
$$

- accuracy is "the stationarity of the conformity degree of the finished product parameters to the parameters specified in the drawing of the product" [6] or "preservation of the parameters of manufactured products within acceptable limits during the manufacture of the entire batch" [7], - accuracy coefficient (2)

$$
k_{a c c}=\frac{6 S}{\delta} ;
$$

- attunement is the constancy-in-time of the correspondence of the average value of a parameter to a given value of the midpoint of the tolerance range [7] - coefficient of attunement (3):

$$
k_{\text {mid }}=\frac{\bar{Y}-Y_{\delta}}{\delta} ;
$$

Where $S, S_{t 1}, S_{t 2}$ are standard deviation of the cumulative sample and private samples at times $t 1$ and $t 2$;

$\bar{Y}, Y_{\delta}$ are average value and midpoint of the tolerance range respectively;

- reliability is "the property ... to keep in time, within the established limits, the values of all parameters characterizing the ability to perform the required functions ..." [8].

In the future, in order to avoid ambiguity, to determine the constancy of the characteristics of the process in time, we will use the term of stability.

The above interpretations assume a certain static nature of the technological process both the average value and the random scatter of the values of the controlled quantity are considered unchanged over time. At the same time, it is assumed as an axiom that the distribution of values in each particular sample obeys the law of normal distribution [6]. It is well known that such a statement is true in relation to the geometric accuracy of dimensions, obtained, as a rule, by certain methods of machining in large-scale or mass production. However, the recommended statistical criteria and so-called threshold values of stability estimates [4 - 8] are determined specifically for the law of normal distribution.

It will be shown hereinafter that methods for quantitatively assessing stability can be considered regardless of the nature of the quality criterion being assessed - i.e., dimensional accuracy, shape accuracy, micro-profile parameters, etc. - for various distribution laws.

Note that the recommendations of regulatory documents are applicable for processes with a clearly pronounced stochastic nature; variations in the quantitative indicators of the result of process implementation occur mainly randomly. It is the account of the stochasticity of the processes that underlies the consideration of the Gauss law as the distribution law of the analyzed values. This approach does not take into account the features of machining processes, for which a certain correlation can be identified between input factors (technological modes, etc.) and output indicators (product quality parameters, process performance, etc.).

\section{Stability of partially deterministic processes}

As far as machining processes with a pronounced deterministic nature (Fig. 1) are considered from the positions mentioned hereinabove, it is impossible obtaining reliable results since a significant deviation of the result obtained from a given average value will be interpreted as a loss of process stability. 


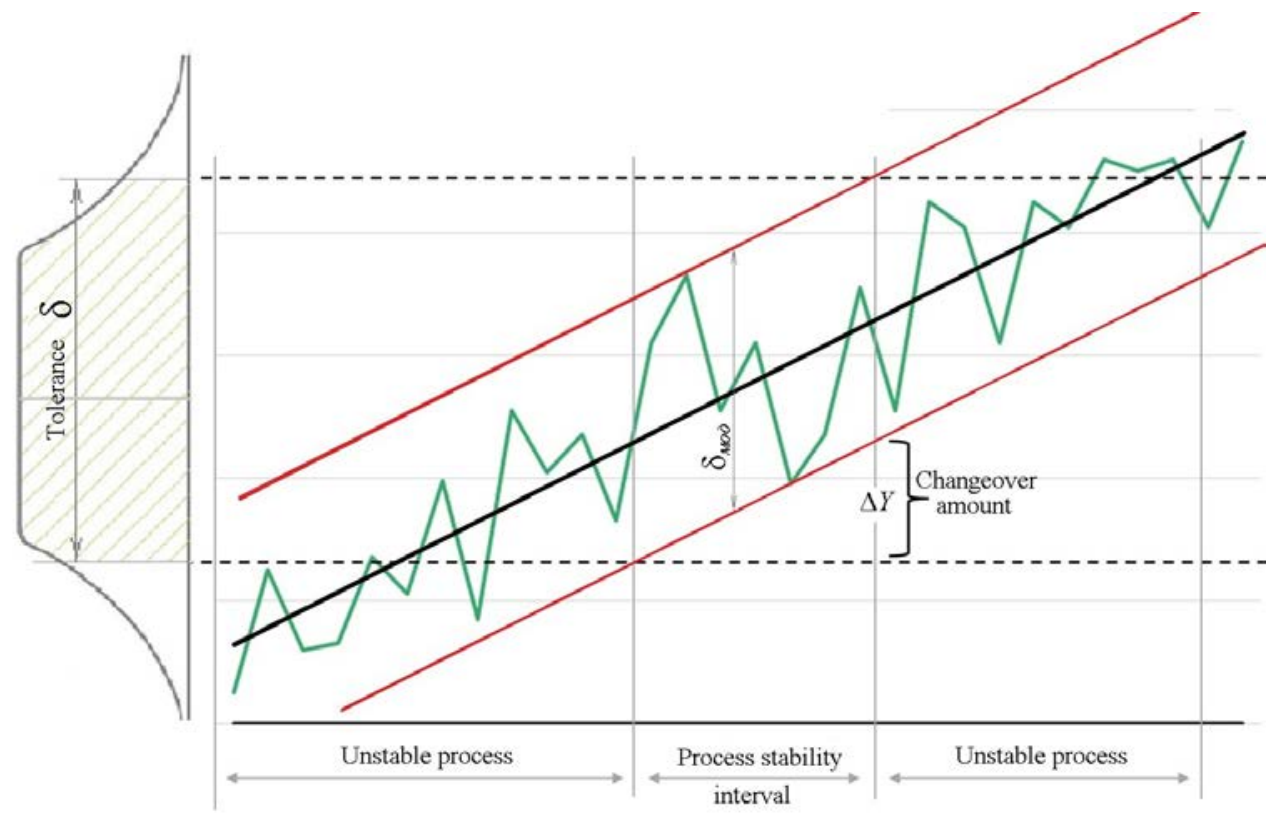

Fig. 1. An example of a partially deterministic process

The stability of a partially deterministic process does not mean that the random variability is greater, less, or appropriate. It should be said that variability can be predicted using statistical methods. The recommendations for assessing accuracy and stability [6] and for using statistical methods [9] implicitly proposes the concept of a statistically controlled process, i.e., a process, "each quality indicator of which is in a state of statistical control" [6] or a process, "in which there are no signs of specific causes of variation" [9].

The "special reasons" for variations are due to the violations of the technical system. A far as recommendations $[6,9]$ in regard to machining processes are dealt with, a reasonable change in the machining conditions for the purpose of predicting the impact on the result (e.g., when using an adaptive control system) will be considered as a special cause of variation (system malfunction). As a consequence, such a process should be referred to unstable.

Let us take as a working definition the following: - a partially deterministic process is statistically controlled if there is a statistically reliable mathematically described regularity [10] between the input parameters of the process and the output ones.

As indicators of the quality of such a process (Fig. 1), not only static characteristics (mean value, standard deviation $\mathrm{S}(\mathrm{Y})$ for the sampling population) but also dynamic (regularly changing mean, standard error of model S) are considered [11, 12]. A quantitative assessment of stability, in this case, can be considered as the Fisher criterion [13]. Earlier in [14], we described in detail an example of assessing the stability of a statistically controlled process.

The mathematically described regularity between the input and output parameters of the process can be obtained, for example, by the methods of regression analysis [11 - 13] in the form of a model $Y=F(t)$ (Fig. 2). The use of regression analysis for modeling complex stochastic processes, such as machining processes, can be considered appropriate $[10,13]$. 


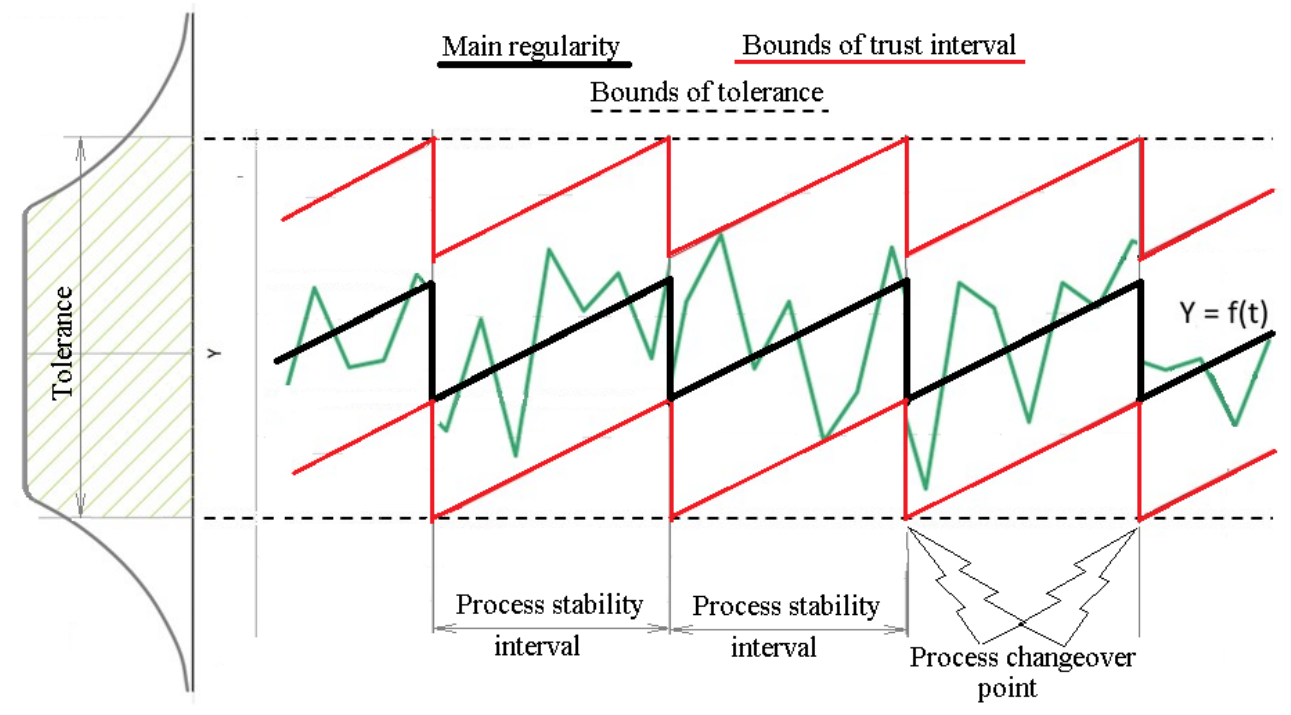

Fig. 2. Scheme of a stable statistically controlled process

In this case, we are considering a one-factor model. The time parameter $(t)$ is chosen as an independent variable, as it is the most common for any process [12]. The regression model should be considered statistically significant if the following condition (4) is met:

$$
S_{\text {mod }}=\sqrt{\frac{\sum_{i=1}^{n} Y_{i}-F\left(t_{i}\right)^{2}}{n}} \leq \sqrt{\frac{\sum_{i=1}^{n}\left(Y_{i}-\bar{Y}\right)^{2}}{n}}=S,
$$

where $S_{\text {mod }}, S, \bar{Y}$ - standard error of the model $Y=F(t)$, standard deviation and mean value of the aggregate sample respectively;

$Y_{i}, n-\quad$ each value and size of the aggregate sample.

The probabilistic assessment of the statistical significance $\alpha(5)$ is determined by the value of the Fisher criterion $[12,13]$.

$$
F_{(1-\alpha)}(n-1 ; n-1)=\left(\frac{S}{S_{\text {мод }}}\right)^{2} \text {. }
$$

In accordance with the concept of accuracy [1, 6, 7], a partially deterministic process is stable in the time interval (Fig. 1), during which the scattered field of the controlled value is within a given tolerance. In the case of a monotonically increasing function, the beginning of the stability interval is determined by the intersection of the lower boundary of the scattered field with the lower boundary of the tolerance field. The moment of completion of the stability interval comes respectively when the upper boundary of the scattered field coincides with the upper boundary of the tolerance. Outside the scattered interval, the entering of the controlled value into the tolerance field is random and does not change the unstable state of the process.

Knowing the moments of the beginning and end of the stability time interval, it is possible to calculate the value of the process adjustment:

$$
\Delta Y=\delta-\delta_{\text {мод }} .
$$

The calculated correction value $\Delta \mathrm{Y}(6)$ is entered into the process settings at certain points (Fig. 2) of the adjustment. The statistically controlled process is graphically displayed in the form of a 'sawtooth' curve, completely within the specified tolerance 
(together with the scattering field). The scattering field width of the controlled parameter is determined by traditional methods $[4,9,10,12,13]$, e.g., according to the confidence level or in accordance with the six-sigma rule.

Checking the statistically controlled process (Fig. 2) under traditional criteria (1-3) shows that stability is ensured over the required period of time, e.g., during the life of the cutting tool.

\section{Conclusion}

- In regard to the processes and technologies of machining, there is no single concept stability fixed in regulatory documents. The concepts of reliability, accuracy, and mood have similar semantic content; and for each concept, original methods of quantitative assessment are recommended.

- The calculation methods and criteria recommended by the current regulatory documents are for use in large-scale or mass production conditions since at the present time the predominant method of organization is small-scale production; in the conditions of modern machining production, traditional criteria for assessing stability, as a rule, are not acceptable.

- The numerical characteristics recommended for assessing the stability of the technological process do not take into account the possibility of a regular change in the average value; when conducting statistical analysis, all errors are considered random; evaluation of processing stability on equipment with adaptive control systems gives incorrect results. In modern conditions, the processes of machining and accordingly the processes of forming the quality of products should be considered as statistically controlled.

\section{References}

1. Suslov, A. G. Design and technological support of quality and competitive ability of engineering products // Science Intensive Technologies in Mechanical Engineering. 2017. - No. 7 (73). - p. 25-28.

2. Technologist's Handbook / ed. by A. G. Suslov - Moscow, Russia : Innovative Mechanical Engineering Publisher, 2019. - pp. 800.

3. Quality management systems. Fundamentals and vocabulary. National Standard R ISO 9000-2015. (2019) Standardinform Publishing. Moscow, Russia.

4. Statistical methods. Process Capability Indicators. Basic calculation methods State Standard R 50779.44-2001. (2001) Standard Publishing. Moscow, Russia.

5. Statistical methods. Statistical quality management. Terms and Definitions. State Standard R 50779.11-2000 (ISO 3534.2-93) (2008) Standardinform Publishing. Moscow, Russia.

6. Recommendations for assessing the accuracy and stability of technological processes. R 50-601-20-91. (1991) Scientific Research Institute for Standardization. Moscow, Russia.

7. Goryachev, V. V. and Kherson, N. S. To the question of assessing the accuracy of technological processes. (2010) Methods of quality management. $7: 56-58$.

8. Reliability in technology. Basic concepts. Terms and Definitions. USSR State Standard 27.002-89. (2002). Standard Publishing. Moscow, Russia.

9. Recommendations for Standardization. Statistical methods. Examples of application. 
Part 8. Statistical process control. R 50.1.087-2013. (2014) Standardinform Publishing. Moscow, Russia.

10. Statistical Methods from the Viewpoint of Quality Control. Shewhart, W.A. (1939, reprint 1986)- N.Y., Dover Publications, Inc. URL : https://deming.pro/assets/statistical-method-from-the-viewpoint-of-quality-controlshewhart-walter.pdf.

11. Kristal, Mark G. and Gorelova, A. Yu. (2019) Processing of the results of planning an extreme experiment : tutorial. Volgograd, Russia

12. Chigirinskaya, N. V., Tchigirinsky, Ju. L. and Gorobtsov, A. S. (2020) Modeling nonperiodic stochastic processes : tutorial. Volgograd, Russia

13. Ronald A. Fisher (1954). Statistical Methods for Research Workers (12th ed.). Edinburgh: Oliver and Boyd. 978-0-05-002170-5. ISBN: 978-0-05-002170-5

14. Tchigirinsky, Ju. L., Evtyunin, A. V. and Kharlamov, V. O. Mechanical treatment's stability quantitative assessment (2021) Bulletin of Volgograd State Technical University, Series "Progressive Technologies in Mechanical Engineering". 1 (248): 4144. 